\title{
Brainstem Glioblastoma Multiforme in a Patient with NF1
}

\author{
JOHN T. FORTUNATO ${ }^{1}$, BRIAN REYS $^{2}$, PAWAN SINGH $^{3}$ and EDWARD PAN ${ }^{3}$ \\ ${ }^{1}$ Henry Ford Hospital, Detroit, MI, U.S.A.; \\ ${ }^{2}$ UT southwestern Simmons Comprehensive Cancer Center, Dallas, TX, U.S.A.; \\ ${ }^{3}$ UT Southwestern Medical Center, Dallas, TX, U.S.A.
}

\begin{abstract}
This case report presents the first known case of a brainstem glioblastoma multiforme (GBM) in a patient with neurofibromatosis type 1 (NF1). While research has proposed that larger germ-line mutations in NFI may be the driving factor that predisposes patients with NF1 to high-grade astrocytomas, this patient had a nonsense mutation in the NFI gene, suggesting a variant tumorigenesis. Limited data on targeted immunotherapy for NF1 patients with a GBM have been reported and more data are required before targeted therapies could be proven as second-line treatment options.
\end{abstract}

While associations between neurofibromatosis type 1 (NF1) and CNS tumors, such as optic pathway gliomas and low-grade brainstem gliomas are well established, the prevalence of glioblastoma multiforme (GBM) in NF1 is less common (1-3). However, patients with NF1 are at a 10- to 50-fold increased risk of developing high-grade gliomas compared to those without NF1 (4). Typically, GBMs in NF1 patients are treated with concurrent radiotherapy and temozolomide (TMZ), which is standard of care for sporadic GBM patients $(5,6)$.

Few cases of NF1-associated GBMs have been reported. NF1-associated GBMs have been reported in the frontal lobe $(5,7)$, occipital lobe $(8)$, parietal lobe $(9)$, cerebellum $(10$, 11 ), and thalamus (12). We present the case of a 23 year-old male with NF1 and a midbrain GBM. To our knowledge, none of the NF1-associated GBMs have been reported in the brainstem.

\section{Case Report}

A 23 year-old male with NF1 presented with 3 months of progressively worsening dizziness, vertigo, headaches,

Correspondence to: John T. Fortunato, Henry Ford Hospital, 2799 W Grand Blvd, Detroit, MI, 48202, U.S.A. Tel: +1 4192613700, e-mail: john.fortunato22@gmail.com

Key Words: Glioblastoma multiforme, neurofibromatosis type 1 , immunotherapy, brainstem. increased sleep, slurred speech, and confusion. He also had double vision for 3 months, requiring a right eye patch. A postcontrast brain magnetic resonance imaging (MRI) showed a ring enhancing mass lesion in the midbrain (Figure 1).

Biopsy demonstrated a grade IV astrocytoma (GBM) that was IDH wild-type, H3K27 negative, and had ATRX loss. FoundationOne $C$ genetic testing of the tumor tissue revealed genetic alterations of the $N F 1$ gene c. $3628 \mathrm{G}>\mathrm{T}$ (E1210*) and c. 4234A $>\mathrm{T}$ (R1412*), ATRX gene 569delC (P190fs*16), and $C D K N 2 A / B$ copy number loss. The $N F 1$ alterations were both nonsense mutations; c. $3628 \mathrm{G}>\mathrm{T}$ was identified at an allele frequency of $51.5 \%$ in the tumor, suggestive of germ line origin.

The patient received concurrent radiotherapy plus oral TMZ for 6 weeks followed by 12 cycles of adjuvant TMZ. The patient also elected to receive treatment with Optune. He subsequently developed significant steroid side effects as well as adrenal insufficiency.

\section{Discussion}

Brainstem gliomas that occur in patients with NF1 are typically low-grade, indolent, and generally have a better prognosis compared to patients with sporadic brainstem gliomas (13-15). Further, most of these lesions are discovered in childhood $(1,4,16)$. High-grade astrocytomas, while rare, have been reported in NF1, but are almost always supratentorial $(5,7-9,12)$. Two cases of infratentorial GBMs in patients with NF1 have been reported, both occurring in the cerebellum $(10,11)$.

Molecular analysis of NF1-related low- and high-grade astrocytomas showed $N F 1$ inactivation in all NF1-associated astrocytomas (12). Molecular analysis of NF1-associated GBMs has shown both an NF1 deletion and either CDKN2A loss or homozygous chromosome 10q losses, which are commonly seen mutations in sporadic GBMs. Since both low-grade and high-grade astrocytomas in NF1 have NF1 inactivation, but only the latter has additional tumor markers that are commonly seen in GBMs, it is suspected that their respective tumorigenesis occurs via distinct pathways. 

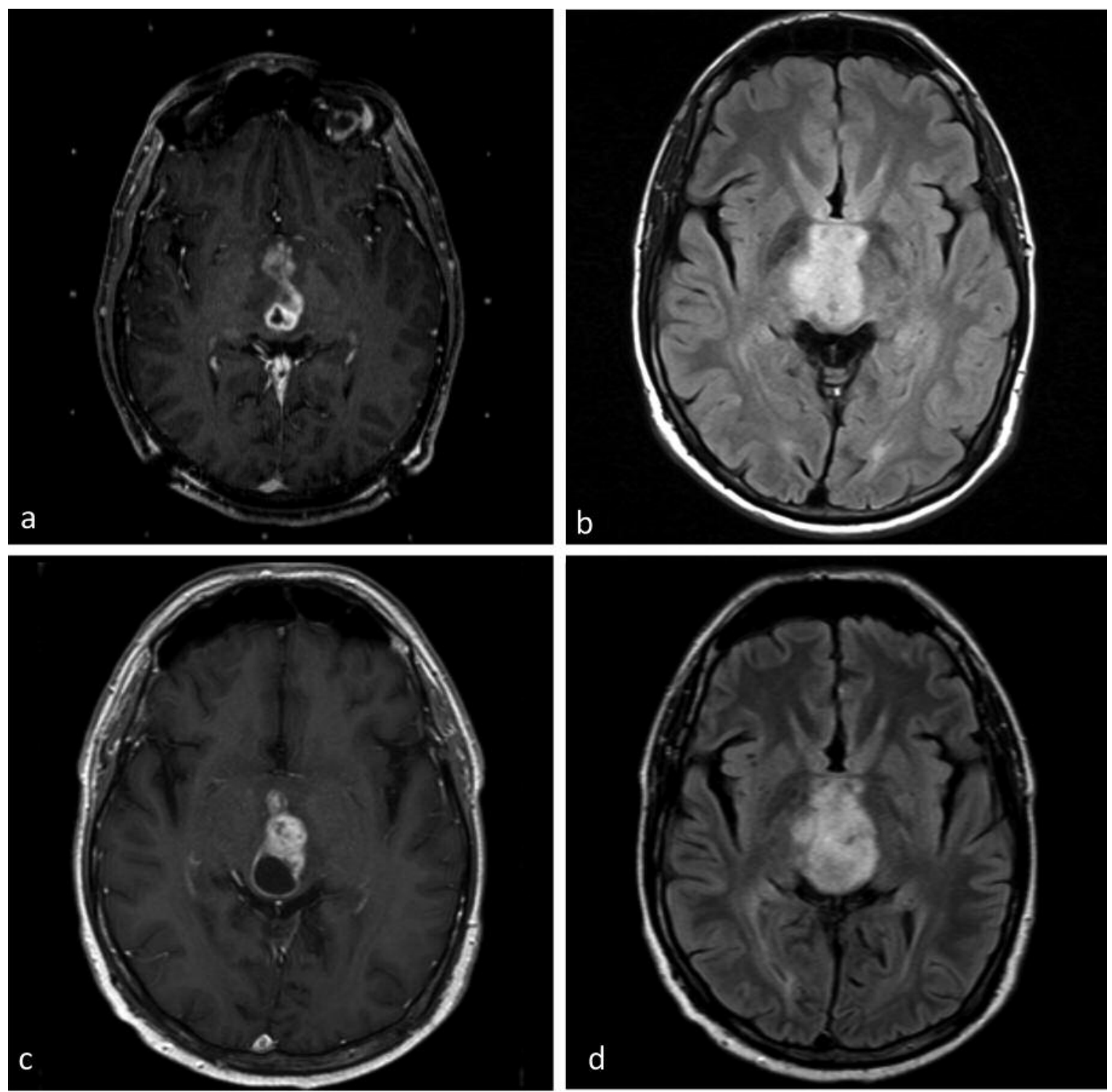

Figure 1. Brain MRI before and after treatment. A: Three months pre-treatment axial T1 post-contrast. B: Three months pre-treatment axial Flair. C: 5 months after treatment initiation axial T1 post-contrast. D: Five months after treatment initiation axial Flair.

Further, it has been proposed that large germ-line deletions of NF1 may be the driving factor that places patients with $N F 1$ at an increased risk of malignant astrocytomas as opposed to low grade gliomas (12).

Our case has analogous findings of both $N F 1$ inactivation and loss of the $C D K N 2 A$ tumor suppressor gene on chromosome 9. Our case had a nonsense NF1 mutation rather than a large NF1 deletion previously noted in other cases with high-grade astrocytoma. Thus, we propose protein-truncating mutations in NF1 may yield similar behaviors to large $N F 1$ deletions when occurring in conjunction with loss of $C D K N 2 A$.

Biopsy of a cerebellar GBM in 2009 revealed two distinct lesions: (1) a grade 4 astrocytoma (GBM) (+GFAP, - S-100, $30 \%$ immunopositivity for $\mathrm{p} 53$ ) and a diffuse neurofibroma (-GFAP, +S-100). First, this supports previous evidence that mutations in both $N F 1$ and p53 cooperate in the development of astrocytomas $(12,17,18)$. Second, the authors hypothesized that the pre-existing neurofibroma may have created a favorable environment for tumor-initiating cells. 
Thus they recommended close surveillance of existing lesions in NFl for the development of high grade astrocytomas (10). Our patient's GBM had no p53 mutation. Though he refrained from MRI surveillance for the 4 years prior to his diagnosis, he had no previous MRI evidence of an infratentorial lesion; nor did his pathology or molecular reports indicate any separate cell type. Therefore, it is likely that his GBM developed de novo. Regardless, the utility of MRI surveillance for patients with $N F 1$ to monitor for both existing and de novo lesions is further reinforced.

While the molecular profiles of these tumors are of significant interest for the development of targeted treatment, more research is required. To date, treatment for NF1 associated GBMs is identical to treatment for sporadic GBMs (5, 6). Targeted therapies, such as immune checkpoints inhibitors, are currently being assessed in clinical trials and could be beneficial for patients with NF1 associated GBMs (19). Of the cases discussed above, three received surgery followed by concurrent radiation and TMZ followed by monthly adjuvant TMZ. One patient lived 41 months after surgery (frontal GBM), (5) another 6 months after surgery (cerebellar GBM), (10) and the third was still alive at the time of publication (frontal GBM, no progression 9 months after surgery) (7). A separate case of an NF1 associated cerebellar glioblastoma showed substantial improvement on MR imaging after 3 weeks of targeted immunotherapy with trametinib, a MEK inhibitor (11). Genomic testing for this tumor revealed no $I D H-1$ mutation, with $N F 1$ splice site $3870+1 \mathrm{G}>\mathrm{T}, C D K N 2 A / B$. Trametinib was used after failure of the standard radiation and TMZ, carboplatin, and also progression on everolimus, an mTOR inhibitor. It should be noted that trametinib was chosen not based on a specific MEK amplification, but because of potential over-activity of the MEK pathway in NF1 (11). More research is required to determine if additional NF1associated GBM patients would benefit from similar immunotherapy.

\section{Conclusion}

In conclusion, we present a case of an NF1-associated midbrain GBM in young adulthood, which to our knowledge has not been previously reported. While data are limited, the molecular profile of this tumor is similar to other $N F 1$ related GBMs, having both an $N F 1$ mutation and $C D K N 2 A$ loss. More research is required to determine if targeted therapies could provide benefit as second line treatment options.

\section{Acknowledgements}

Dr. Pawan Singh's fellowship was funded by educational grants from AbbVie Pharmaceuticals and Leadiant Biosciences.

\section{References}

1 Guillamo J-S, Créange A, Kalifa C, Grill J, Rodriguez D, Doz F, Barbarot S, Zerah M, Sanson M, Bastuji-Garin S, Wolkenstein $\mathrm{P}$ and Réseau NF France: Prognostic factors of CNS tumours in Neurofibromatosis 1 (NF1): a retrospective study of 104 patients. Brain J Neurol 126(Pt 1): 152-160, 2003.

2 Créange A, Zeller J, Rostaing-Rigattieri S, Brugières $P$, Degos JD, Revuz J and Wolkenstein P: Neurological complications of neurofibromatosis type 1 in adulthood. Brain 122(Pt 3): 473$481,1999$.

3 Campian J and Gutmann DH: CNS tumors in neurofibromatosis. J Clin Oncol Off J Am Soc Clin Oncol 35(21): 2378$2385,2017$.

4 Gutmann DH, Rasmussen SA, Wolkenstein P, MacCollin MM, Guha A, Inskip PD, North KN, Poyhonen M, Birch PH and Friedman JM: Gliomas presenting after age 10 in individuals with neurofibromatosis type 1 (NF1). Neurology 59(5): 759-761, 2002.

5 Hakan $\mathrm{T}$ and Aker FV: Case report on a patient with neurofibromatosis type 1 and a frontal cystic glioblastoma. Neurol Neurochir Pol 42(4): 362-365, 2008.

6 Stupp R, Mason WP, van den Bent MJ, Weller M, Fisher B, Taphoorn MJB, Belanger K, Brandes AA, Marosi C, Bogdahn U, Curschmann J, Janzer RC, Ludwin SK, Gorlia T, Allgeier A, Lacombe D, Carincross JG, Eisenhauer E and Mirimanoff RO: Radiotherapy plus concomitant and adjuvant temozolomide for glioblastoma. N Engl J Med 352(10): 987-996, 2005.

7 Jeong TS and Yee GT: Glioblastoma in a patient with neurofibromatosis Type 1: A case report and review of the literature. Brain Tumor Res Treat 2(1): 36-38, 2014.

8 Pál E, Gömöri EE and Gáti I: Neurofibromatosis and glioblastoma in a case of multiple sclerosis. Eur J Neurol $8(6)$ : 717-718, 2001.

9 Abraham M, Ennis P and Mehta R: Glioblastoma multiforme in an adult patient with von recklinghausen disease. Community Oncol 5(10), 2008. doi: 10.1016/S1548-5315(11)70509-5. [Epub ahead of print].

10 Broekman MLD, Risselada R, Engelen-Lee J, Spliet WGM and Verweij BH: Glioblastoma multiforme in the posterior cranial fossa in a patient with neurofibromatosis type I. Case Rep Med 2009: 757898, 2009.

11 Ameratunga M, McArthur G, Gan H and Cher L: Prolonged disease control with MEK inhibitor in neurofibromatosis type Iassociated glioblastoma. J Clin Pharm Ther 41(3): 357-359, 2016.

12 Gutmann DH, James CD, Poyhonen M, Louis DN, Ferner R, Guha A, Hariharan S, Viskochil D and Perry A: Molecular analysis of astrocytomas presenting after age 10 in individuals with NF1. Neurology 61(10): 1397-1400, 2003.

13 Pollack IF, Shultz B and Mulvihill JJ: The management of brainstem gliomas in patients with neurofibromatosis 1 . Neurology 46(6): 1652-1660, 1996.

14 Molloy PT, Bilaniuk LT, Vaughan SN, Needle MN, Liu GT, Zackai EH and Phillips PC: Brainstem tumors in patients with neurofibromatosis type 1: a distinct clinical entity. Neurology 45(10): 1897-1902, 1995.

15 Ullrich NJ, Raja AI, Irons MB, Kieran MW and Goumnerova L: Brainstem lesions in neurofibromatosis type 1 . Neurosurgery 61(4): 762-766, 2007. 
16 Mahdi J, Shah AC, Sato A, Morris SM, McKinstry RC, Listernick R, Packer RJ, Fisher MJ and Gutmann DH: A multiinstitutional study of brainstem gliomas in children with neurofibromatosis type 1. Neurology 88(16): 1584-1589, 2017.

17 Zhu Y, Guignard F, Zhao D, Liu L, Burns DK, Mason RP, Messing A and Parada LF: Early inactivation of p53 tumor suppressor gene cooperating with NF1 loss induces malignant astrocytoma. Cancer Cell 8(2): 119-130, 2005.

18 Reilly KM, Loisel DA, Bronson RT, McLaughlin ME and Jacks T: Nf1; Trp53 mutant mice develop glioblastoma with evidence of strain-specific effects. Nat Genet 26(1): 109-113, 2000.
19 Polivka J Jr., Polivka J, Holubec L, Kubikova T, Priban V, Hes $\mathrm{O}$, Pivovarcikova $\mathrm{K}$ and Treskova I: Advances in experimental targeted therapy and immunotherapy for patients with glioblastoma multiforme. Anticancer Res 37: 21-24, 2017.

Received May 31, 2018

Revised June 22, 2018

Accepted June 25, 2018 